\title{
Simulation of the Electron Flux into the Main Injector Electron Cloud Retarding Field Analyzer Using Vorpal
}

\author{
Paul L.G. Lebrun ${ }^{*}, 1$, James F. Amundson ${ }^{1}$, Panagiotis G. Spentzouris ${ }^{1}$, Seth A. Veitzer ${ }^{2}$ and Peter Stoltz ${ }^{2}$ \\ ${ }^{I}$ Fermi National Accelerator Laboratory, Illinois, USA \\ ${ }^{2}$ Tech-X Corp., Boulder, Colorado, USA
}

\begin{abstract}
We present results from precision simulations of the electron cloud (EC) in the Fermilab Main Injector (MI), focusing on the dynamics of the EC close to the Retarding Field Analyzer (RFA) detectors. Our simulations are based on the Vorpal plasma simulation package. We find that the presence of a parasitic, weak (few Gauss) magnetic field significantly alters the spatial distribution of electrons in the cloud. The detected flux can easily change by factors of two depending on the location of the RFA. Moreover, the growth rate of the EC is also sensitive to such magnetic fields. Therefore, we suggest to (i) upgrade the RFA preamplifier to $\approx 10 \mathrm{MHz}$ bandwidth such that the change in EC growth rate during a complete MI bunch train can be detected; (ii) install a small solenoid (of $\approx 25$ Gauss maximum) to optionally switch off the EC, and thereby cleanly establish the presence of low energy electrons in the pipe; and (iii) design an RFA that can be inserted in $\mathrm{a} \approx 2 \mathrm{kGauss}$ field, such that an EC signal can be seen in the environment that matters, i.e., in the MI dipoles.
\end{abstract}

PACS Numbers: 29.27.-a.

Keywords: Accelerator, instrumentation, simulation, particle-in-cell, electron-cloud.

\section{INTRODUCTION}

The electron cloud (EC) phenomenon in high intensity proton storage rings and synchrotrons can limit the performance of such machines $[1,2]$. This phenomenon is characterized by an exponential growth of the number of low energy $(\mathrm{eV})$ electrons emitted at the surface of the beam pipe wall. The initial seed for the uncontrolled growth of the electron density comes from ionization of the residual gas in the beam pipe by the high energy proton beam. These electrons are then accelerated by the field induced by the passing proton beam. This radial electric field could reach tens of $\mathrm{kV} / \mathrm{m}$ at a few millimeters from the proton bunch centroid. The resulting acceleration for electrons in the beam region is sufficient to propel these electrons towards the beam pipe walls with kinetic energy commensurate with the threshold for secondary emission. The resulting low energy secondary electrons are then accelerated by the field of the next proton bunch, causing more secondary emission. Note that the proton current also creates a small (fraction of a Gauss) magnetic field, leading to non radial trajectories for low energy electrons. The EC density eventually stabilizes due to the intense space charge close the beam pipe wall. However, the electric field induced by this moving cloud of electrons can be sufficient to create significant disruption of the proton beam.

An essential goal of our numerical models is to interpret and guide further experimental developments. More specifically, our focus here is to provide valuable information

*Address correspondence to this author at the Fermi National Accelerator Laboratory, Illinois, USA; Tel: 1-630-840-3947;

Fax: 1-630-840-6039; E-mail: lebrun@fnal.gov regarding the interpretation of data taken with Retarding Field Analyzers (RFAs) [3, 4] at the the Fermilab Main Injector (MI). An RFA is basically a Faraday cup capable of collecting ballistic electrons. The resulting current is measured at a frequency of a few $\mathrm{Hz}$. To avoid runaway or unstable currents, a grid located upstream of the collector has a bias voltage of a minimum of 20 Volts. Only electrons with kinetic energies above $20 \mathrm{eV}$ will be collected by such a device. The RFA is mounted on the outer side beam pipe. Small holes or slits in the pipe allow the device to sample the moving electron cloud. The simple sketch shown in Fig. (1) illustrates the experimental setup.

In the MI electron cloud experiment, non-directional magnetometers located in the vicinity of one of these RFAs have been replaced by a new magnetometer that measures each spatial component of the field. Data from this new magnetometer, shown in Fig. (2), has been used in our 3dimensional Vorpal [5] simulations. As we will show, we are able to confirm the sensitivity of this detection technique to small parasitic magnetic fields. This note describes the simulation method and our results as well as giving suggestions on how to verify some of our Vorpal-based predictions.

\section{SIMULATION MODELS OF THE MAIN INJECTOR RFA}

The configuration of the Fermilab MI RFA has been previously described $[3,4]$. Here we limit ourselves to a brief description of the salient parameters of the MI with regards to simulation of the RFA performance. The total length of the MI is about $3.32 \mathrm{~km}$; one turn takes about $11 \mu \mathrm{s}$. The radio frequency system for acceleration and longitudinal control of the proton bunch has a fundamental frequency of $53.1 \mathrm{MHz}$, 
with a bunch spacing of about $18.8 \mathrm{~ns}$. As the injection energy is $8 \mathrm{GeV}$, the protons are relativistic throughout the synchrotron cycle. There are typically 5 continuous trains of 72 bunches, separated by a few empty rf cycles. In addition, there is an abort gap between two of the bunch trains, of a duration of approximately 0.8 microseconds. This specific timing of the bunch train could be of use to diagnose the growth and decay time of the electron cloud, should the instrumentation be fast enough.

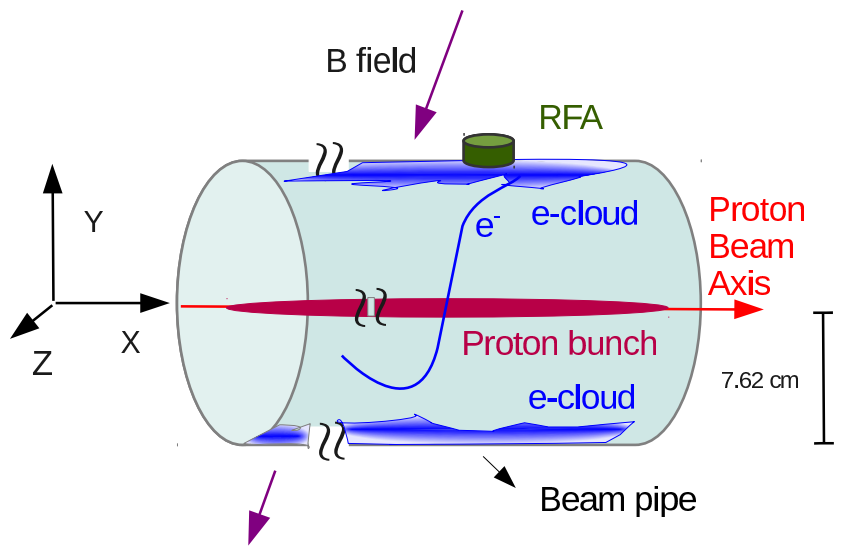

Fig. (1). Sketch of the experimental setup, showing the coordinate system used throughout the calculation, the cylindrical beam pipe, taken as infinitely long in the simulation, the RFA, and the rough direction of the stray magnetic field. The transverse size of the beam is exaggerated in the figure, as the beam radius is only a few $\mathrm{mm}$.

We now focus on the relevant details of Vorpal simulations of the RFA. Vorpal [5], is an object-oriented $\mathrm{C}++$ framework for 1-, 2- and 3-dimensional, parallel electromagnetic and electrostatic simulation of fully relativistic charged fluids and particles on a structured grid. Vorpal includes $2^{\text {nd }}$-order accurate physical models of particle/wall interactions, secondary electron emission, and space charge effects, as well as mechanisms for measuring time histories of all physically relevant quantities.

A 3-dimensional code is required for this application, as the arbitrary direction of the parasitic field breaks both the left/right longitudinal symmetry and the azimuthal symmetry. Also note that the magnetic field created by the beam current, peaking at about 11 A (approximating the bunch shape as square, one ns duration, pulse of $7.0 \times 10^{10}$ protons per bunch), produces a magnetic field at $\approx 1 \mathrm{~cm}$ from the beam that is comparable in magnitude to the parasitic field. The use of Vorpal in this modeling context has been previously documented [6]. The length of the physical region where the EC is simulated is $50 \mathrm{~cm}$ long, which is much larger than the diameter of the pipe (15.24 $\mathrm{cm})$ and the size of the RFA aperture. The secondary emission yield (SEY) is assumed to be 2.2, as the beam pipe is made of un-scrubbed stainless steel ${ }^{1}$.

\footnotetext{
${ }^{1}$ This large SEY value is probably realistic after the pipe has been exposed to air, then pumped down, as is the case in the beginning of a typical run. At the beginning of a run, the RFA signal tends to be relatively high, then, probably due to scrubbing, the signal decreases over time, in a matter of days to weeks.
}

A detailed numerical model of the RFA, with its exact physical geometry, including the field shaping grid, in the presence of the proton beam current has not yet been implemented. Such a detailed simulation is outside of the scope of this work. However, the simpler simulation presented here already provides interesting results. The entrance of the RFA has been simulated with two distinct models. First, the SEY at the entrance slots has been set to zero, such that no electrons are directly produced within the aperture of the device. Second, simulated electrons are tracked so that we can choose electrons entering the RFA that only have an energy greater than some threshold.

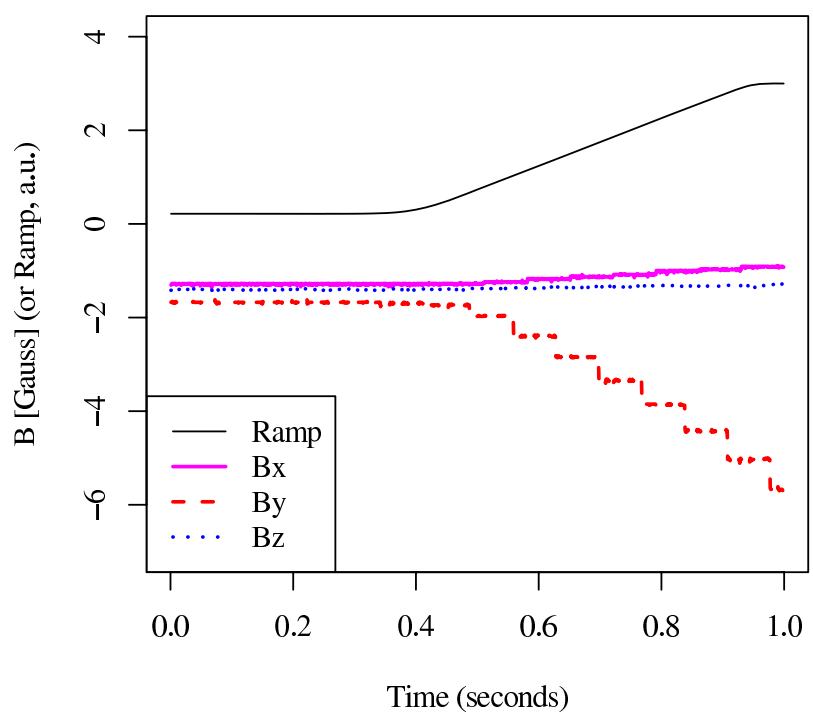

Fig. (2). The parasitic magnetic field as a function of time during the MI ramp (shown in black).

This is a non-relativistic problem, where radiation from the electrons is negligible and where no external radio frequency fields are present. Thus EM radiation can be altogether ignored. However, the field due to the space charge fields in the EC itself are important, and they do change on a time scale commensurate with the passage of the proton beam. The corresponding electric field has a broad frequency spectrum ${ }^{2}$, some of it above the frequency cutoff of the beam pipe $(1.5 \mathrm{GHz})$.

Finally, as in any particle-in-cell (PIC) code, one must be careful to use the appropriate grid size to avoid numerical uncertainties due to poor resolution of relevant features. In our case, for a transverse grid size of $3.4 \mathrm{~mm}$, spikes in the electron density appeared at azimuthal angles $\varphi=0, \pm \pi$ and $\pm \pi / 2$, when stray magnetic fields were turned off and the beam was exactly centered in the cylindrical beam pipe. That is, no asymmetry at all in $\varphi$ would be expected. However, since the electron density varies rapidly in the region within a few $\mathrm{mm}$ from the wall, such a spurious asymmetry, due to artifacts of the PIC algorithms, is not at all surprising. Reducing the transverse cell size to $1.7 \mathrm{~mm}$ mostly fixed the problem, leaving a residual $\varphi$ asymmetry of less then two percent at the beginning of the simulation and $0.5 \%$ after a few proton bunches, when the statistics start to accumulate

\footnotetext{
${ }^{2}$ See, for instance, Figure 11 in Lebrun et al. [6].
} 
due to the onset of cloud buildup. This adjustment of the non-physical parameter of the calculation becomes relevant when a real asymmetry is introduced in the problem, as discussed in the first paragraph of the next section.

\section{SIMULATION RESULTS}

The goal of this particular simulation is to investigate the reliability of the EC densities measured by the RFA in the MI in the presence of systematic uncertainties. More specifically, as the RFA detects only a small fraction of the electrons in the pipe, assumptions about the uniformity of the EC density must be assessed. The first systematic uncertainty could be due to a beam displacement with respect to the center of the beam pipe. Indeed, if the beam is displaced vertically with respect to the beam pipe walls, a $\varphi$ anisotropy appears quite early in the cloud buildup process, at the onset of the exponential growth of the cloud, as shown in Fig. (3). This anisotropy gradually disappears as the electrons have a chance to migrate away from the beam region.

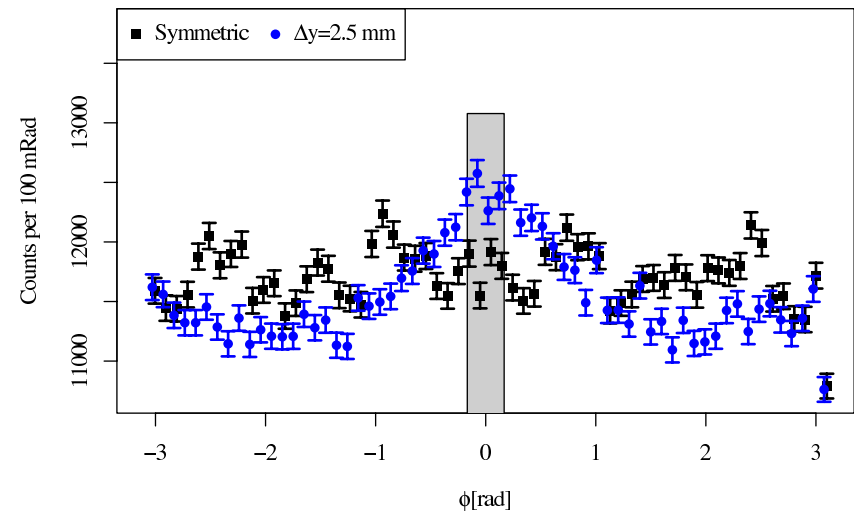

Fig. (3). Distribution of the electrons' azimuthal angle $\varphi$ with respect to the vertical direction ( $\mathrm{Y}$ axis) $128 \mathrm{~ns}$ after the first proton bunch. Electrons located near the upstream/downstream edges of the pipe have been rejected. The electron density is also smooth along the proton beam axis. No cuts have been placed on the electron energy. The darker rectangle indicates the azimuthal angle aperture of the real RFA.

The presence of stray magnetic fields reconfigures the cloud as well, as shown in Fig. (4). At all times during the bunch train, the induced asymmetry in $\varphi$ due to off-axis magnetic fields is larger than that induced by a beam offset.

As expected, this asymmetry depends on the electron energy in a non-trivial way; the trajectories of electrons due to stray magnetic fields, the proton bunch and the space charge of the cloud all depend on both time and space. The asymmetry in $\varphi$ persists at later times, and is more pronounced for more energetic electrons, as shown in Fig. (5). The scatter in the data, which is significantly greater than the expected statistical fluctuations in the number of macro-particles in the simulation, is simply due to the fact that the EC density is sampled every 7.3 ns while the EC is strongly perturbed at every bunch crossing, i.e., every 18.8 ns.

The EC density is probed every $\sim 0.5 \mathrm{~ns}$ during the simulation. The electron density averaged over the physical region ( $\pm 75 \mathrm{~cm}$ of the pipe) is shown in Fig. (6). From this data, we estimate the fast growth time, defined as the duration for one e-folding of this density, during the phase where the cloud density is increasing exponentially. When the self space charge forces dominate the electron motion, namely between proton bunches, electrons have time to move back to the wall, and the exponential growth stops. The electron cloud density reaches saturation in about $500 \mathrm{~ns}$ in this simulation. In reality, such an integrated time scale could be much longer if the rarefied electron cloud generated by gas ionization, as seen by the first proton bunch, is much lower. Thus, such time scales are a non-trivial probe of the dynamics, from the exponential growth phase to the phase where the self space charge forces dominate the motion between proton bunches.
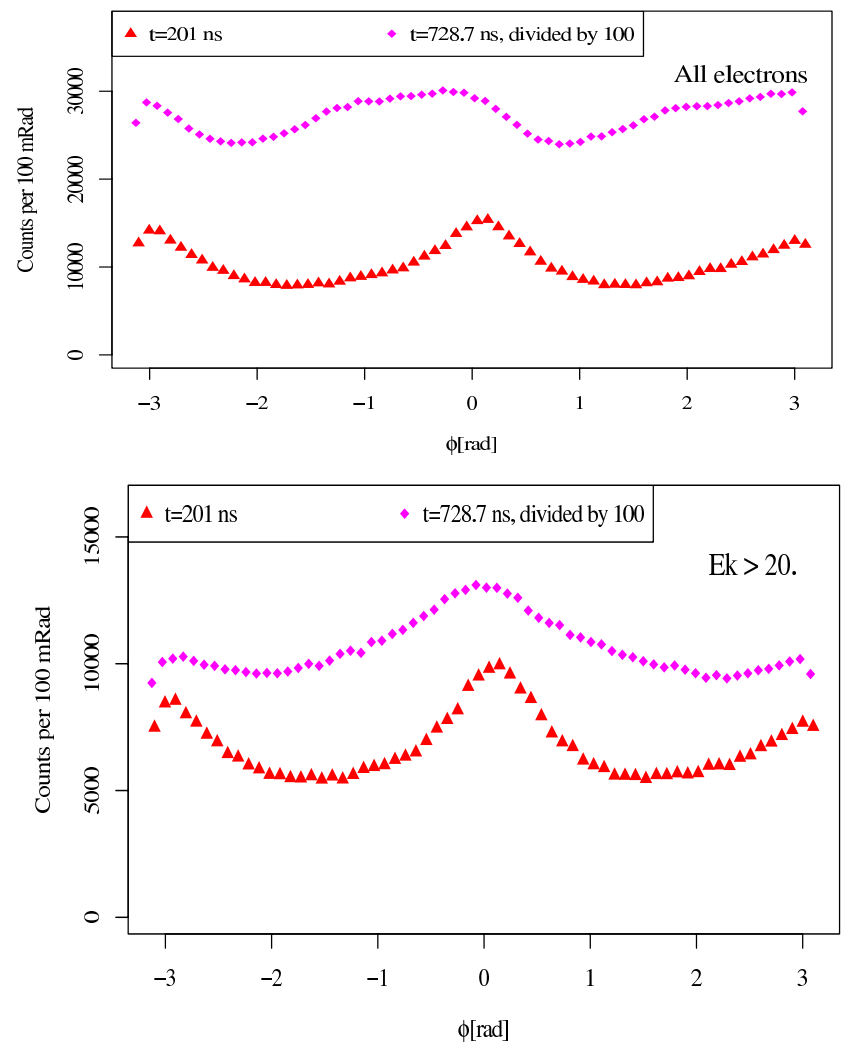

Fig. (4). Top: Distribution of the same electrons' azimuthal angle $\varphi$, taken $201 \mathrm{~ns}$ after the first bunch in the booster batch, and 28 bunches later (at $t=727 \mathrm{~ns}$ ). Note that the density of the EC grows by a factor $\sim 50$ between these times. At $t=201 \mathrm{~ns}$, the density of the EC is not saturated, while at $t \approx 727 \mathrm{~ns}$, when space charge dominates the dynamics between proton bunches, a more uniform distribution of electron charges appears. Bottom: Same as above, but for all macro-electrons whose energy is greater than $20 \mathrm{eV}$. This azimuthal asymmetry is a bit more pronounced.

Note that the Larmor radius in the stray magnetic field is often smaller than the radius of the pipe. Consequently, this stray magnetic field allows for a slightly more efficient transport and acceleration of the electrons from the beam region to the beam pipe wall, provided that the field's orientation is perpendicular to the surfaces of this wall.

For the relevant case, where a stray magnetic field is present, the EC is in a nearly steady state after about 30 proton bunch crossings. The density reaches $2.4 \times 10^{12}$ 
electrons $/ \mathrm{m}^{3}$, corresponding to a ratio of the electron to proton linear density of about $50 \%{ }^{3}$. The EC density above a kinetic energy of $20(40) \mathrm{eV}$ is about $5 \times 10^{11}\left(2.3 \times 10^{11}\right)$ electrons $/ \mathrm{m}^{3}$, respectively.

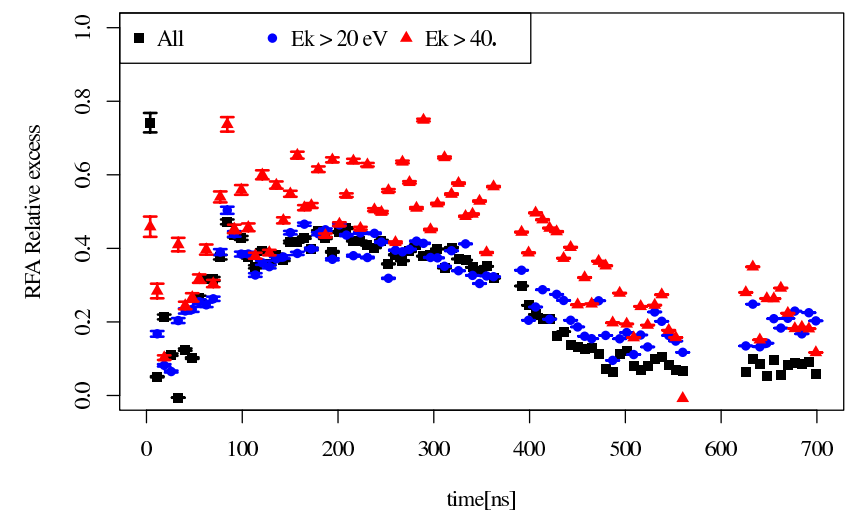

Fig. (5). Evolution of the $\varphi$ asymmetry, defined as the integrated density within the $\varphi$ RFA aperture relative to the average density, for macro-particles a few mm away from the entrance of the RFA, over about 30 proton bunches, for three values of the threshold cut on the macro electron kinetic energy. The gap at $600 \mathrm{~ns}$ reflects missing data from simulation and has no physical origin.

From the simulated data, the electron flux into the RFA can be readily estimated. The ensemble of macro-electrons that hits the wall in the vicinity of the RFA has been saved so that a post-simulation analysis can be performed on electrons that might enter the RFA. If integrated over the duration of the passing of a few proton bunches, the exact longitudinal position of such macro-electrons does not matter, allowing us to consider a bigger sample of electrons than the one covering the limited extent of the RFA, which has a physical aperture of about $2.5 \mathrm{~cm}$ in diameter. The distributions of the transverse coordinates are also fairly uniform across the limited aperture of the RFA. The momentum distributions are those simulated by Vorpal. These macro-electrons have been propagated through both the entrance slits of the RFA and the static electric field located between the grid and the collector. The electric field maps are based on a detailed simulation using the [7] software package [3]. The simulated current on the collector is shown in Fig. (7). The slits and the grid are assumed to be completely passive, in the sense that possible field emissions from edges are neglected, as are any possible secondary emissions. The bias voltage on the RFA's grid was set to +20 Volts.

On the time scale of the passing of a few proton bunches, the current is proportional to the density, and saturates at 4.6 $\mu \mathrm{A}$. On shorter time scales, both density and predicted RFA current fluctuate by quite a bit more than the expected statistical uncertainty on the number of macro-particles. This reflects the broad frequency content of the electromagnetic fields present in the cloud.

\footnotetext{
${ }^{3}$ The linear proton density is measured when the bunches are present in the pipe, over an effective length of $\sqrt{2 \pi} \sigma$, or $8.4 \times 10^{10}$ electrons $/ \mathrm{m}$, for $7 \times$ $10^{10}$ protons per bunch.
}
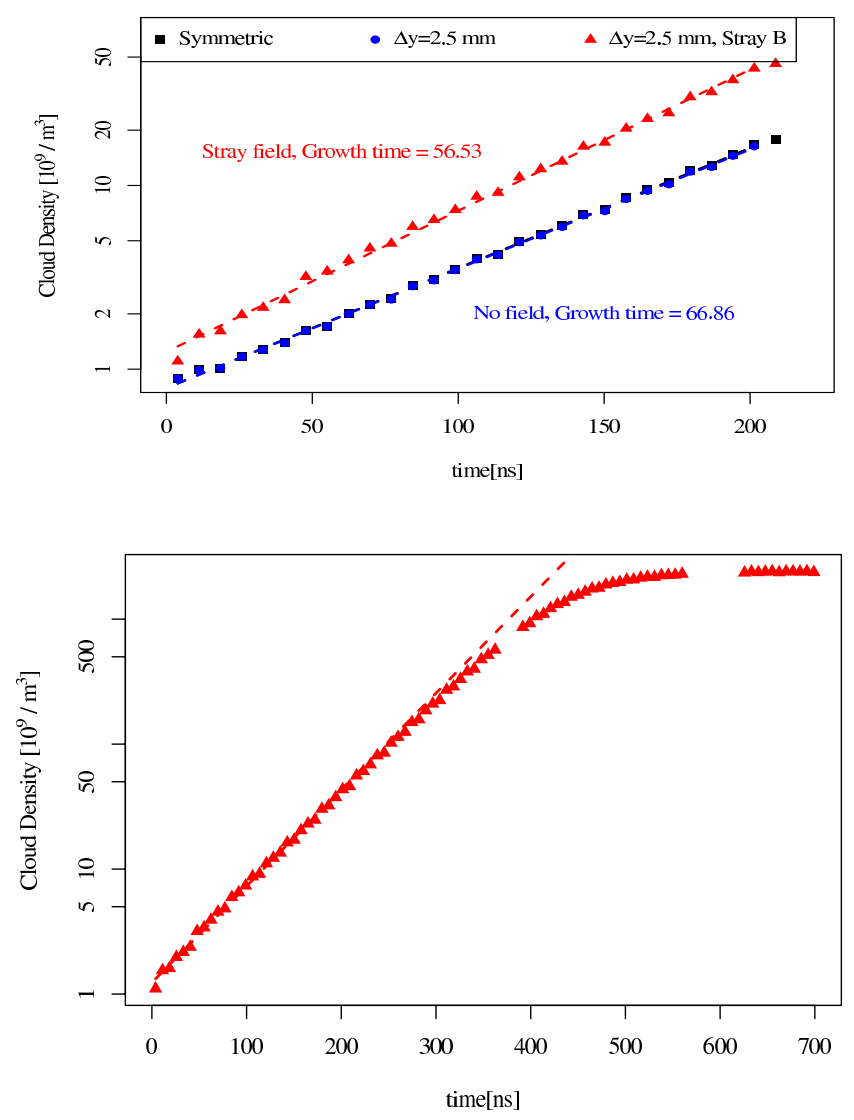

Fig. (6). The EC density as a function of time after the first bunch crossing. Top: during the exponential growth phase, with and without the stray magnetic field. Bottom: over a longer times scale, reaching saturation after about 500 ns. Gaps in the data are due to sampling artifacts and have no physical meaning.

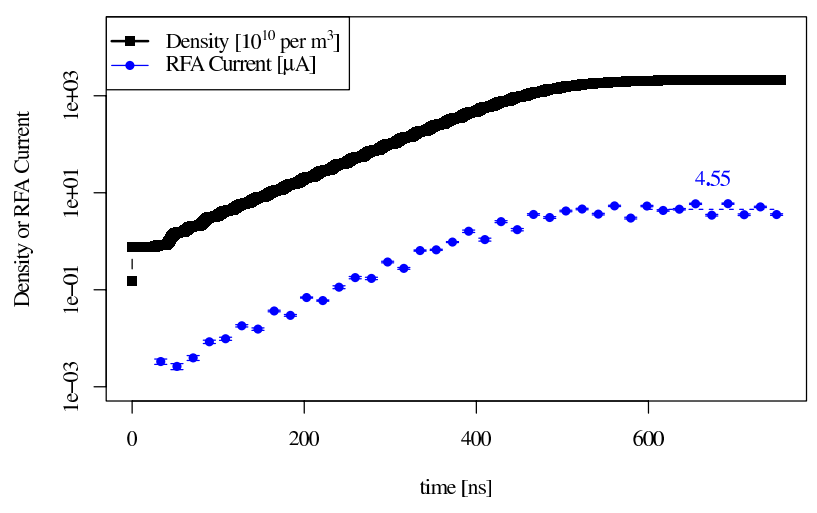

Fig. (7). The simulated electron current measured by the RFA and the cloud density. The stray magnetic field is taken from ref. [2].

A well known electron cloud mitigation method is to install solenoidal fields that are parallel to the beam axis and whose strength is sufficient to confine the electrons to either the center of the pipe or close to the wall [8]. In either case, such electrons are not efficiently accelerated in the electric field generate by the proton bunches, and the exponential growth of the EC does not occur. In our case, our simulations indicate that a longitudinal, extinguishing, field of 25 Gauss is sufficient to prevent the uncontrolled growth of the EC. However, if there exist non-solenoidal fields the 
cloud region, the effectiveness of this field to mitigate the buildup of the cloud is greatly reduced [9].

\section{CONCLUSIONS}

We have performed simulations of electron clouds near the MI RFA. The presence of a stray magnetic field as low as $\approx 3.5$ Gauss affects both the growth time and the geometry of the EC. Noting that real RFA devices can only measure the integrated flux above $\approx 20$ Volt, over many turns, as the response time of the preamplifier is no better than $1.0 / 3.0 \mathrm{kHz}$, many of our predictions can not yet be verified. Nevertheless, it is clear that the current configuration of stray magnetic fields boosts the yield of electrons that the RFA collects. This excess yield should persist up the ramp, as the vertical component of the stray field increases along with the MI ramp. This is not what has been observed during the recent operations of the MI [10]. To resolve this discrepancy and better understand the performance of the device, we suggest the installation of an extinguishing solenoid over a section of $\approx \pm 50 \mathrm{~cm}$ in length. The second suggested upgrade is to improve the bandwidth of the electronics that measure the RFA current, such that near extinction of the cloud in the beam abort gap can be detected. Ultimately, $\approx 5$ ns timing could be achieved with a more involved detector, where electrons produce photons off a scintillating material, and such photons are detected with a fast image amplifier, multi-anode PMTs, or if cost a real concern, a single PMT.

Ultimately, we will have to determine the EC properties in the environment where it really counts, and where a solenoidal field can not be generated: in the dipoles and quadrupole of the synchrotron, which make up over $65 \%$ of the length of the ring. In these magnets, the field is strong and perpendicular to the walls, allowing for efficient transport of electrons from the beam region to the top and bottom sections of the beam pipe (or, inside the quadrupole, at 45 degrees).

\section{CONFLICT OF INTEREST}

The authors confirm that this article content has no conflict of interest.

\section{ACKNOWLEDGEMENTS}

The RFA field map (shown in Fig. 2) was prepared and delivered to us by Chen Yang Tan. We greatly appreciate his contribution. Numerous discussions with Bob Zwaska were very productive.

This work was supported by the United States Department of Energy under contract DE-AC02-07CH11359 and the ComPASS project funded through the Scientific Discovery through Advanced Computing program in the DOE Office of High Energy Physics. This research used resources of the National Energy Research Scientific Computing Center, which is supported by the Office of Science of the U.S. Department of Energy under Contract No. DE-AC02-05CH11231. This research used resources of the Argonne Leadership Computing Facility at Argonne National Laboratory, which is supported by the Office of Science of the U.S. Department of Energy under contract DE-AC02-06CH11357.

\section{REFERENCES}

[1] Zimmermann F. Review of single bunch instabilities driven by an electron cloud. Phys Rev ST Accel Beams 2004; 7: 124801.

[2] Pivi MT, Furman MA. Electron cloud development in the proton storage ring and in the spallation neutron source. Phys Rev ST Accel Beams 2003; 6.

[3] Zwaska R, Tan CY, Duel KL. An improved retarding field analyzer for electroncloud studies. Proceedings of PAC09 2009. Available from: http://accelconf.web.cern.ch/AccelConf/PAC2009/papers/th5 rfp041.pdf

[4] Zwaska R, Tan CY, Backfish M. The ecloud measurement setup in the main injector. 2010. Available from: http://www-spires.sl ac.stanford.edu/spires/find/hep/www? $\mathrm{r}=$ fermilab-conf-10-508-ad

[5] Cary JR, Nieter C. Vorpal: a versatile plasma simulation code. J Comp Phys 2004; 196: 448-73.

[6] Lebrun P, Veitzer SA, Stoltz P, Spentzouris PG, Cary JR. Simulation of the electron cloud in the fermilab main injector. 2011. Available from: http://inspirebeta.net/record/913965

[7] SIMION. Industry standard charged particle optics simulation software. 2008; 1. Available from: http://simion.com

[8] Wang JQ, Guo ZY, Liu YD, Qin Q, Xing J, Zhao Z. Electron instability studies in the beijing electron positron collider. Phys Rev ST Accel Beams 2004; 7: 094401.

[9] Veitzer SA, Lebrun P, Cary JR, Spentzouris P, Stoltz PH, Amundson JF. Computation of electron cloud diagnostics and mitigation in the main injector. J Phys Conf Ser 2009; 180: 012007. DOI: 10.1088/1742-6596/180/1/012007.

[10] Zwaska R. Electron cloud experiments at fermilab: formation and mitigation. 2011. Available from: http://ss.fnal.gov/archive/2011/ conf/fermilab-conf-11-081-apc.pdf 